\title{
Review Article \\ Epithelial-Mesenchymal Transition in Pancreatic Cancer: A Review
}

\author{
Shuai Wang, Shuai Huang, and Yu Ling Sun \\ Institute of Hepatobiliary and Pancreatic Diseases, School of Medicine, Department of Hepatobiliary and Pancreatic Surgery, \\ The First Affiliated Hospital of Zhengzhou University, Zhengzhou University, Zhengzhou, China \\ Correspondence should be addressed to Yu Ling Sun; ylsun@zzu.edu.cn
}

Received 16 July 2017; Revised 21 October 2017; Accepted 19 November 2017; Published 12 December 2017

Academic Editor: Stephen H. Safe

Copyright (C) 2017 Shuai Wang et al. This is an open access article distributed under the Creative Commons Attribution License, which permits unrestricted use, distribution, and reproduction in any medium, provided the original work is properly cited.

\begin{abstract}
Pancreatic ductal adenocarcinoma (PDAC) is one of the most aggressive solid malignancies and is characterized by its insensitivity to current therapy. The invasion and metastasis of solid tumors such as PDAC are complex processes involving many factors. Recent insights into the role of cancer stem cells (CSCs) and the epithelial-mesenchymal transition (EMT) in tumorigenesis have increased the knowledge base and highlighted new therapeutic targets of this disease. The process of EMT is regulated by a complex network of cytokines, transcription factors, growth factors, signaling pathways, and the tumor microenvironment, exhibiting CSClike properties. The transition of solid cancer cells from an epithelial to a mesenchymal phenotype increases their migratory and invasive properties, thus promoting metastasis. In PDAC, the exact influence of EMT on the biological behaviors of cancer cells and its impact on clinical therapy remain controversial, but the therapeutic strategy of combining EMT inhibition with chemotherapy deserves attention. Alternatively, anti-inflammatory therapy that targets the interaction between inflammation and EMT is a valid strategy for treating the premalignant stage of tumor progression. In this review, we summarize the latest research on EMT and the potential relationship between EMT and PDAC.
\end{abstract}

\section{Introduction}

Pancreatic ductal adenocarcinoma (PDAC), a serious global health burden, is the eighth most common cause of cancerrelated death worldwide. PDAC has an extremely poor prognosis, with a 5-year overall survival rate of less than $5.0 \%[1,2]$. In China, the median survival time of PDAC patients is approximately 7.8 months. Among PDAC patients, $30.0 \%$ receive curative radical operations, whereas only $9.8 \%$ undergo comprehensive effective treatment [3] due to the controversy regarding the fundamental causes of the disease. The low survival rate of PDAC can be attributed to an aggressive biological phenotype that is characterized by early local invasion and metastasis $[2,4,5]$. Accordingly, there is an urgent need to elucidate the molecular mechanisms associated with the occurrence, development, therapeutic resistance, and metastasis of this lethal disease. Numerous studies have demonstrated that the invasiveness of pancreatic cancer correlates with the epithelial-mesenchymal transition (EMT) [6-9].
EMT is a morphologic cellular program simply defined as the phenotypic transition from an epithelial to a mesenchymal state. In vivo, intermediate hybrid epithelial and mesenchymal phenotypes are frequently observed and are referred to as partial EMT. The mesenchymal phenotype is considered "metastable," whereas the epithelial phenotype is thought to be stable and capable of colonization. EMT is modulated by complex regulatory networks involving epigenetic modification and transcriptional control, including EMT-inducing transcription factors (EMT-TFs) such as SNAIL (Zinc finger protein SNAIL), ZEB (Zinc finger E-boxbinding homeobox 1), and Twist (Twist Basic Helix-LoopHelix Transcription Factor 1) and transcription regulators, such as microRNAs (miRNAs). Regarding metastasis, numerous studies have shown that most circulating tumor cells express both epithelial and mesenchymal markers, emphasizing the crucial role of EMT during carcinoma dissemination [10-12]. Furthermore, studies have demonstrated that loss of SNAIL or ZEB, which are strong epithelial EMT repressors, in pancreatic cells does not prevent PDAC 


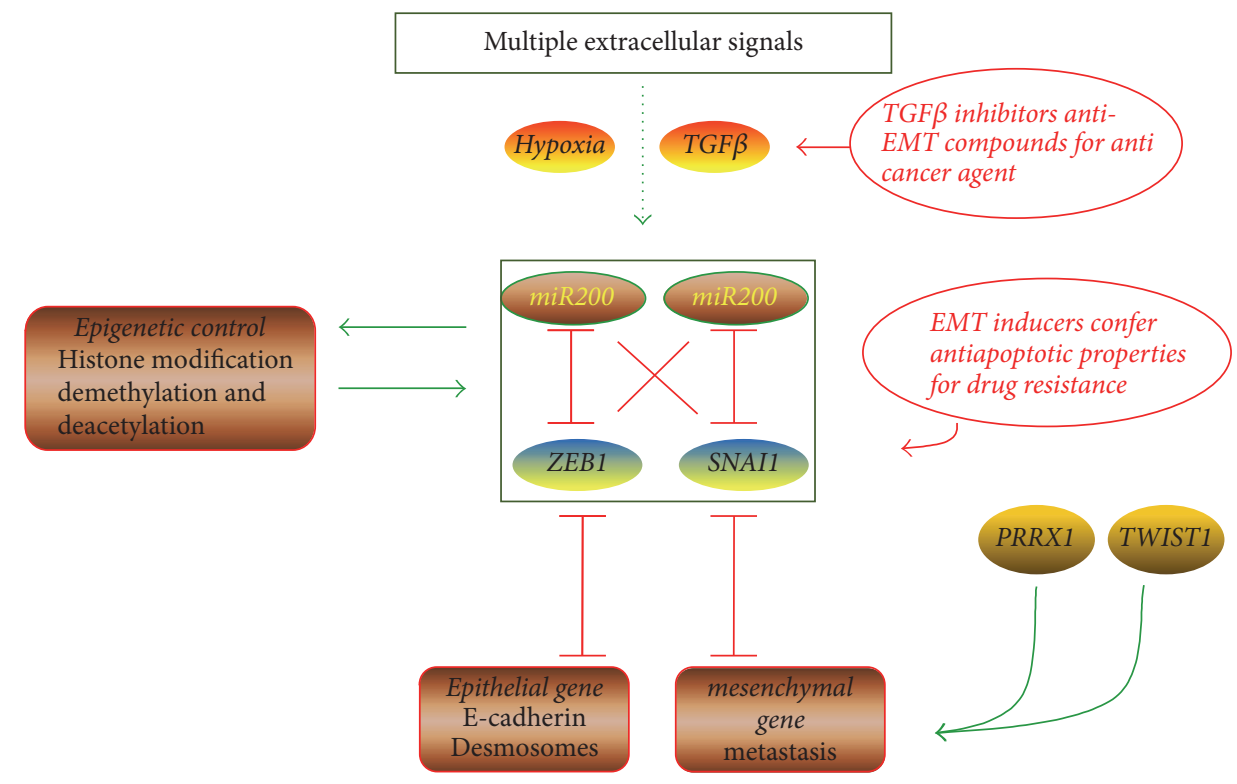

FIGURE 1: Regulatory network for EMT: key sites conferring chemoresistance as well as anti-EMT agents for cancer therapy.

metastasis, thus opposing the classical notion of EMT [13]. EMT occurs at the tumor invasive front, which is functionally different from the bulk of tumor $[14,15]$ and shows cancer stem cell (CSC) involvement [16]. Moreover, correlations between some EMT-TFs associated with pancreatic carcinoma and stemness have been verified because TFs such as ZEB1, a stemness repressor that inhibits miRNA, often promote stemness. Similar to metastasis and CSCs, EMT exhibits a significant association with resistance to chemotherapy.

In this review, we discuss the roles of EMT in the dissemination and metastasis of pancreatic cancer, explore the relevance of CSCs in the tumorigenesis, colonization, and metastatic processes, and discuss the role of the inflammation environment in promoting EMT in premalignant lesions. We also discuss potential therapies and patient selection criteria based on chemotherapy according to the association between EMT and chemoresistance with the aim of improving the prognosis of patients with pancreatic cancer.

\section{EMT Regulation}

EMT is regulated by a complex network involving epigenetic modifications, transcriptional control, alternative splicing, protein stability, and subcellular localization [17-19]. Some pathways might be crucial for a given EMT event during tumor progression such as differentiation, metastasis, and tumorigenesis. Members of the transforming growth factor$\beta$ (TGF- $\beta$ ) superfamily have been implicated as major EMT induction signals during almost all morphogenetic events, including tumorigenesis and metastasis [20]. EMT-TFs, such as SNAIL, ZEB, and Twist, are considered master regulators of these complex networks (Figure 1). In addition, multiple miRNAs, including miR-200 and miR-34, establish a negative feedback loop to maintain epithelial and mesenchymal homeostasis [17, 21, 22].
TGF- $\beta$ signaling is considered a primary EMT inducer, although the precise pathways activated by individual family members may differ during different morphologic events, some of which include direct phosphorylation by ligandactivated receptors of SMADs [SMA (small body size) and MAD (mothers against decapentaplegic) related proteins] TFs and by certain cytoplasmic proteins regulating cell polarity and tight-junction formation [23]. TGF- $\beta$ also influences multiple other EMT-inducing signal transduction pathways, involving Notch, Wnt (wingless (wg)/int (proto-oncogene integration-1) related genes), and integrin signaling pathways, which act in concert to trigger EMT [24].

The EMT-TFs SNAIL and ZEB are defined as potent epithelial repressors rather than mesenchymal promoters, whereas PRRX and Twist are considered strong mesenchymal inducers. SNAIL and ZEB repress the expression of epithelial markers, for example, E-cadherin, claudins, and occludins, which are downstream of this regulatory loop. Due to the "metastable" mesenchymal phenotype, the aforementioned EMT-TFs are important metastasis promoters, especially ZEB [25]. The miR-200 family is incongruously associated with both reduced invasion [26, 27] and increased metastasis [28] - which resulted in combined suppression of the EMTpromoting transcription factors SNAIL1/2, Twist, ZEB1, and ZEB2 but had no effect on metastasis. In turn, ZEB1 represses expression of the miR-200 family, including the stemnessinhibiting miRNA miR-203. This negative feedback highlights the importance of EMT-TFs in stemness and the relevance of EMT regulation and stemness maintenance to pancreatic tumors. In addition, PRRX1 (Paired Related Homeobox 1), an EMT inducer in the SNAIL1-independent pathway, drives invasiveness together with TWIST1 [29].

EMT can be regulated by upstream and downstream epigenetic changes, largely affecting the EMT miRNA-SNAILZEB networks, as these EMT transcriptional drivers are 


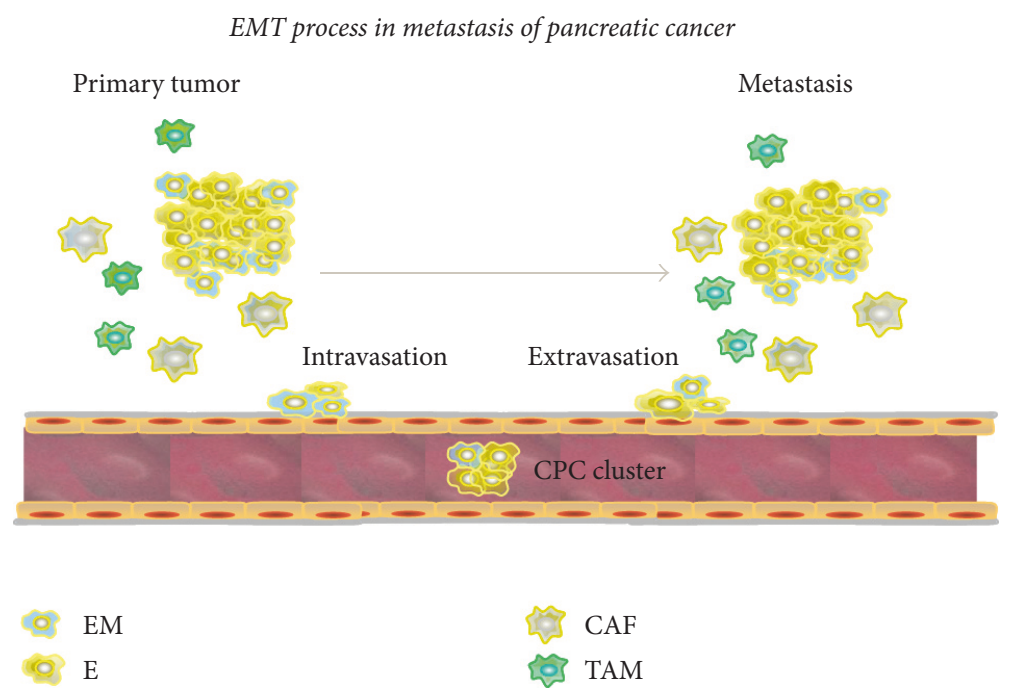

FIGURE 2: EMT during the metastatic process of pancreatic cancer. Circulating tumor cells (CTCs) or circulating pancreatic cells (CPCs) derived from primary tumor cells in pancreatic cancer undergo EMT and transition to hybrid phenotypes at the invasive front. CPC clusters colonize a distant site via the circulation, where, according to some studies, the cluster undergoes the reverse of EMT. EM: the hybrid phenotype; E: epithelial phenotype; CAF: cancer-associated fibroblast; TAM: tumor-associated macrophage.

involved in a complex epigenetic regulatory loop [30]. The major mechanisms of epigenetic regulation consist of histone modifications, DNA methylation, and X-chromosome inactivation [31, 32]. Simultaneous with EMT progression, cells in the tumor invasive front differentiate into an invasive subtype, and the epigenome of these cells is continually undergoing demethylation and deacetylation. In this process, SNAIl activates chromatin modifiers such as lysine-specific demethylase 1 (LSD1) [33], G9a (euchromatic histone lysine methyltransferase 2) and Suv39H1 (suppressor of variegation 3-9 homolog 1) histone methyltransferases [34, 35], SIN3A (SIN3 transcription regulator family member A), histone deacetylase 1 (HDAC1), and HDAC2 [36], and ZEB1 participates by recruiting the LSD1-containing corepressor complex, $\mathrm{HDACl}$ and HDAC2 [37]. miR-200 family expression is also mediated by histone demethylase and DNA methylation [38]. Expression of different TF combinations caused by altered histone acetylation marks an essential relevant phenotypic change during EMT $[39,40]$, and EMT-TFs contribute to pluripotency by controlling chromatin and nuclear organization to modulate the transcription of downstream EMT genes.

Further research into the regulation network might provide insight into promising treatments for fatal tumors, especially pancreatic cancer.

\section{Roles of EMT in Pancreatic Cancer Metastasis}

The role of EMT in metastasis is a longstanding source of controversy, largely due to an inability to monitor transient and reversible EMT phenotypes in vivo. The hybrid phenotype, termed partial EMT, has the potential to promote or reverse EMT and is referred to as "metastable" [41]. Metastasis consists of dissemination and colonization, and, via EMT, circulating tumor cells play a vital role in this process. As numerous data have indicated, the proportion of carcinoma cells undergoing EMT in primary tumors is low $[42,43]$; however, most CTCs exhibit both epithelial and mesenchymal traits, indicating that EMT might occur during dissemination of carcinoma cells, including pancreatic cancer cells [10-12]. In the presence of high concentrations of TGF$\beta$-associated platelets, CTCs might originate from carcinoma cells that undergo EMT in the primary tumor or from intermediate EMT phenotypes in circulation. In addition, CTCs display a full range of EMT phenotypes, indicating that these cells express ECM components. The relative enrichment of mesenchymal markers in these cells is indicative of their successful colonization of distant organs, confirming increased abilities to invade and intravasate, although this occurs rarely in pancreatic cancer [44]. At a distant site, CTCs transitioning to the mesenchymal phenotype comprise distinct clonal carcinoma cells held together through intercellular adhesion, emphasizing the requirement for mesenchymal cancer cells to revert to the epithelial state for metastatic growth (Figure 2).

Nevertheless, untransformed epithelial cancer cells can reach distant organs, such as the lung, after traveling to the bloodstream via extravasation. Because epithelial cells, including cell clusters, can efficiently disseminate and extravasate, the EMT/MET pathway might be indispensable in all cells for secondary neoplasm generation [45]. In breast cancer, a mesenchymal-specific Cre-mediated fluorescent marker switch system in spontaneous breast-to-lung metastasis models is used to monitor transient and reversible EMT phenotypes in vivo. The research confirms that inhibiting EMT by overexpressing miR-200 did not impact lung metastasis development [42]. Moreover, studies have investigated the role of EMT in PDAC by generating mouse PDAC models with deletions of SNAIL or Twist, two key EMT transcription factors suppressed by miR-200 family, and the results showed 
that EMT suppression does not block the development of invasive PDAC, systemic dissemination or metastasis [13]. To some degree, these results contradict the necessity for the original EMT/MET hypothesis during tumor progression [41, 46, 47]. Based on cooperation between multiple EMTTFs, it is possible that the loss of individual factors may not prevent cell invasion and dissemination, particularly in pancreatic cancer, in which even normal cells without strong epithelial properties are present [48]. Furthermore, there is evidence that cells in the primary tumor undergo EMT [49].

Intriguingly, invasiveness and dissemination to the bloodstream occur very early during EMT, even before a malignant tumor can be detected. Cells from pancreatic intraepithelial neoplasia (PanIN) lesions, the premalignant lesions that undergo EMT, exhibit invasive traits in vitro [6]. Lineage tracing has suggested that pancreatic cells can delaminate from PanIN lesions and cross the basement membrane, which encases circulating pancreatic cells (CPCs), before such invasive behavior is detected and lesions have seeded to distant organs. CPCs are derived from acinar cells undergoing EMT, and these cells acquire survival and self-renewal properties called stem cell-like characteristics that could be considered tumor-initiating properties. These results are consistent with the finding that because of metastatic disease, the mortality of patients who receive surgical resection of small pancreatic neoplasms with clear surgical margins and no evidence of metastasis is over $75 \%$ within 5 years [50]. Additionally, a fraction of patients who receive a pancreatectomy for chronic pancreatitis with PanIN develop metastatic PDAC. Furthermore, inflammation facilitates EMT, and an increase in the number of CPCs was observed in Pdxl-Cre; Rosa $^{\text {YFP }}$ and Pdx1-Cre; Kras ${ }^{\text {G12D }}$; Rosa ${ }^{\text {YFP }}$ mouse pancreatitis models [6], to some degree, which could prove the significant role inflammation plays in the PDAC metastasis.

Indeed, EMT might facilitate invasion and intravasation of other cells that retain their epithelial characteristics, and it plays a crucial role in early metastasis, even at the premalignant lesion stage. Nonetheless, some authors have questioned whether EMT is a prerequisite for metastasis, especially for colonization [13].

\section{Cancer Stem Cells Undergo EMT in Pancreatic Cancer}

Considering the above data, cells undergoing EMT appear to acquire stem-like features, indicating the crucial role of EMT in CSC generation. CSCs, a small subgroup of tumorigenic cells within tumors, can self-renew, differentiate, survive under stress, and metastasize. CSCs are often identified using a number of cell surface markers, including CD44, CD24, and CD133, which are essential for detecting CSCs in a cluster undergoing EMT $[51,52]$. CSCs, which express traits of both stem cells and cancer cells, have been identified in tumors [53], and based on cell division symmetry and gene expression alterations, CSCs differ from other cells within the tumor [54]. These findings emphasize the crucial role of EMT in cancer recurrence and metastasis.

EMT-TFs, including members of the SNAIL and ZEB families, play crucial roles in the gland-reconstituting activity of stem cells as master regulators [55]. ZEB1 is described as an EMT inducer and transcriptional repressor that in particular represses stemness-inhibiting miR-203 and miR200 family members. Downstream miR-200 family members, including Sox2 (sex determining region Y-Box 2) and Klf4 (Kruppel-like factor 1), are also stem cell factors. Additionally, the correlation between ZEB1 and stemness is measured by the extent of sphere formation in undifferentiated pancreatic cancer cells [25]. For example, ZEB1 knockdown results in poor initiation of sphere formation and a further reduction in sphere number after in vitro migration, indicating that ZEB1 is necessary for self-renewal. Pancreatic CSCs are defined as a CD24+/CD44+ subpopulation [56], and decreased CD24 expression in ZEB1-knockdown cells has been confirmed. However, Snaill mediates a shift from asymmetric (one stem cell, one differentiating cell) to symmetric (two stem cells) cell divisions, showing that EMT has a role increasing CSC numbers in the tumor stem cell reservoir [57]. TGF- $\beta$ is defined as the main and crucial EMT inducer during cancer pathogenesis progression [23] and dramatically enhances the program by which cancer-associated fibroblasts (CAFs) increase the frequency of tumor-initiating cells in cancer patients [53].

Nevertheless, there are numerous data for the uncoupling of EMT and CSCs. According to some evidence, EMT and CSCs can be defined as in parallel pathways rather than in the same pathway, especially during metastatic colonization. It is widely accepted that disseminated tumor cells (DTCs) undergo MET to transition toward a more epithelial phenotype for colonization, and this process is regulated by metastasis-associated macrophages (MAMs) that suppress EMT-TFs [58]. By converting hepatic stellate cells into myofibroblasts, MAMs also play an essential role sustaining metastatic tumor growth [59]. Regarding EMT-TFs, the lack of PRRX1, an EMT promoter, induces the proliferation of clusters and results in mammosphere formation and the emergence of a CD44-high cell cluster, which is considered a stem cell subpopulation [29]. Furthermore, fibroblast reprogramming is also required for EMT during the induction of tumor-initiating capacities. Oct-4 (octamer-binding protein 4), Klf4, c-Myc (Myc proto-oncogene), and Sox2 sequentially induce a high rate of pluripotency [60], and, as mentioned previously, Klf4 and Sox 2 are stem cell factors. Due to such sequential induction, epigenetic reorganization is thought to occur during the interval between stemness and EMT [61].

Intriguingly, the upregulation of SNAIL1 expression in prostate and bladder cancer cells suppresses stemness, which contrasts with other relevant data associating EMT and stemness [62] and resembles the lethal EMT process induced by TGF- $\beta$ in PDAC [63]. In TGF- $\beta$-sensitive PDAC cells, EMT becomes lethal by changing TGF- $\beta$-induced Sox 4 from a tumorigenic inducer to an apoptosis promoter, evidence that supports the uncoupling of EMT and stemness, especially in PDAC.

However, an intermediate EMT state might help to enrich for cells that are prone to exhibiting CSC-like traits, which has been observed in pancreatic cells. The intermediate phenotype derived from epithelial cells undergoing partial EMT displays both epithelial and mesenchymal traits, providing an essential condition for dissemination and colonization [11]. 
MAP3K4/CBP (mitogen-activated protein kinase 4) activity represses epithelial gene expression, causing TS cells to transition to an intermediate EMT state while maintaining their stem cell properties [15, 39], similar to observations in pancreatic cancer cells. Transplantation of $\mathrm{YFP}^{+} \mathrm{E}-\mathrm{cad}^{+}$ or $\mathrm{YFP}^{+} \mathrm{E}$-cad ${ }^{-}$pancreatic cells from PDAC mice into pancreata results in histologically similar tumor formation. Additionally, all of these cells expressed either ZEB1 or Ecad at comparable levels in both groups, indicating EMT progression. Thus, the E-cad status appears to be important for tumorigenesis or stemness. In other words, there is significant plasticity between the epithelial and mesenchymal states considered to be partial EMT [6].

\section{Inflammation Promotes EMT in PanIN}

As mentioned previously, EMT-TF-miRNA networks, for example, the CSC/ZEB1-miR-200 feedback loop, not only explain the mechanism of EMT but also demonstrate its correlation with cancer progression. Because TGF- $\beta$ plays a role in inflammation, the TGF- $\beta$ pathway and resulting inflammation have been implicated in EMT.

Inflammation plays decisive roles at different stages of tumor development, including initiation, promotion, malignant conversion, invasion, and metastasis [64]. In the primary tumor boundary, the persistent recruitment of immune cells, such as macrophages and lymphocytes, is considered a necessary event for establishing the inflammatory tumor microenvironment. Additionally, remarkable interactions between tumor-associated macrophages (TAMs) during tumor cell dissemination and invasion indicate a correlation with EMT $[65,66]$. In pancreatic cancer, inflammation is defined as a significant event during carcinogenesis and premalignant lesion progression $[67,68]$. Additionally, a strong correlation between chronic pancreatitis and pancreatic cancer is now widely accepted [69].

By increasing the number of circulating cancer cells via EMT, inflammation facilitates invasion and dissemination. Additionally, CPCs are known to play a critical role during the metastatic progression of pancreatic cancer and premalignant lesions. CPCs are derived from acinar cells undergoing EMT, which are considered the initial tumor cells; thus, CPCs in circulation might indicate EMT at the primary tumor site. Recently, acute pancreatitis was induced using cerulean and pancreatic duct ligation in KCY mice. As expected, this treatment resulted in the formation of acinar-to-ductal metaplasia with inflammation (ADMIs), PanIN, and defects in epithelial characteristics. Moreover, compared with the source pancreas and sham-treated group, numerous $\mathrm{CD} 24^{+} \mathrm{CD} 44^{+} \mathrm{CPCs}$ (defined as possessing stemness qualities) were detected and strongly increased in the circulation, which is consistent with the observations from the natural PanIN condition. For further verification, posttreatment with dexamethasone resulted in almost complete elimination of PanIN lesions in the pancreas and CPCs in the circulation, highlighting a crucial prerequisite for maintaining PanIN lesions. These data demonstrate that expression of oncogenic Kras facilitates EMT and dissemination in response to acute pancreatitis.
At the molecular level, SNAIL is an essential EMT-TF that is stabilized in the EMT process by the inflammatory cytokine TNF- $\alpha$ (tumor necrosis factor alpha) via activation of the NF$\mathrm{kB}$ (nuclear factor kappa B) pathway [70]. TAMs are essential inflammatory cells that secrete proinflammatory cytokines as TNF- $\alpha$ and facilitate angiogenesis and tissue remodeling, thus promoting tumor cell motility. Additionally, TNF- $\alpha$ activates the major inflammatory response pathway NF-kB, which facilitates both tumor development and metastatic progression [71, 72]. Because SNAIL itself is defined as a repressor of the epithelial phenotype and an inhibitor of apoptosis, the migration of cells undergoing inflammationinduced EMT increases dramatically. Furthermore, the antiinflammatory cytokine TGF- $\beta$ is an important regulator of EMT [23], and numerous studies have demonstrated that the TGF- $\beta$ pathway collaborates with the Wnt, Notch, and MAPK (mitogen-activated protein kinase) pathways to facilitate EMT during various morphological processes. Additionally, the TGF $\beta /$ Smad pathway has been shown to coordinate with Ras activation to promote EMT [73]. However, as the inflammatory microenvironment increases the likelihood of mutations, which are considered to play crucial roles in tumorigenesis, TGF- $\beta$ suppresses epithelial cell proliferation and early tumor growth, causing some tumors to acquire inactivating mutations in TGF- $\beta$ signaling components. Overall, the effectiveness of anti-inflammatory agents in reducing mortality bolsters the association between inflammation and tumor progression [74].

In general, if inflammation promotes EMT by stabilizing SNAIL expression and the TGF- $\beta$ signaling pathway, then anti-inflammatory therapy is a valid strategy for treating the premalignant stage of tumor progression. Furthermore, the number of CPCs might be included as a prognostic predictor for early stage therapy.

\section{EMT and Chemoresistance in Pancreatic Cancer}

Due to a lack of early detection methods, patients are typically diagnosed at a late stage, with a five-year survival rate of $<5 \%$. Surgical resection remains the only curative treatment, but fewer than $20 \%$ of patients qualify as candidates. Chemical therapies have comprised the major available regimens in the last two decades. Unfortunately, the current chemical treatment for advanced PDAC, including gemcitabine-based combinations, molecular target therapy, and multiagent regimens, still results in a poor prognosis, with only $5.2 \%$ of patients surviving after three years [1, 2]. Although gene expression profiling has been applied to identify biomarkers and therapeutic targets in pancreatic cancer [75], the molecular mechanisms of chemoresistance in pancreatic cancer still require elucidation, and EMT might be the crucial event. Various pathways present in the cancer environment, such as chemotherapy resistance, confer resistance to death in cells undergoing EMT [76]. Although the ability of EMT to confer chemoresistance to pancreatic cancer cells is clear, cells undergoing EMT have a limited contribution to tumor progression, including during the establishment of metastases 
and tumorigenesis $[13,76]$. Furthermore, a more "mesenchymal" phenotype exhibits higher drug resistance [77].

Immunotherapy for cancer treatment has generated substantial interest in recent years, with notable successes in several tumor types, including PDAC [2, 78, 79]. Recent studies have elucidated the essential role EMT plays in immunotherapy resistance [80-82]. Immunotherapy, which uses monoclonal antibodies against targets such as programmed death1 (PD1) or cytotoxic $\mathrm{T}$ lymphocyte-associated antigen 4 (CTL4), two inhibitory T-cell receptors, has become prevalent in recent years. As expected, dominant mutations are induced by these therapies; however, these mutations do not contribute to the resistance of CTL cancer cells to lysis, and the EMT of target cancer cells actually abolishes it [83]. Controversially, cancer cells undergoing EMT might not be susceptible to CTL lysis, which has been supported by research in melanoma cells [81]. The cancer cells that undergo SNAIL-mediated EMT are considerably more metastatic than their parental cells, and this has been attributed to the emergence of T regulatory $\left(\mathrm{T}_{\text {reg }}\right.$ ) CD4 cells expressing Foxp3 (Forkhead Box P3), which induces immunosuppression in these cells [81]. Despite a lack of evidence for pancreatic cancer cells, the aforementioned factor might participate in $\mathrm{T}_{\text {reg }}$ cell chemoresistance to drugs such as cyclophosphamide. Intriguingly, tumors that respond best to CTL-A4, and more recently to PD1 and PD1L antibodies, exhibit a higher EMT score, which indicates an increase of target cell tension potentiates killing by CTLs [84]. This research reveals an unappreciated physical dimension to lymphocyte function and demonstrates that cells use mechanical forces to control the activity of outgoing chemical signals, which has been proved in melanomas and renal, bladder, and NSCL carcinomas. There is little evidence to support this phenomenon in PDAC. Further research regarding the interaction between EMT and immunosuppression is needed. In addition to immunotherapy, vaccines are being evaluated in the adjuvant setting for pancreatic cancer; 5-Fluorouracil (5-FU)-based chemoradiation combined with a pancreatic cancer vaccine of irradiated granulocyte-macrophage colony stimulating factor (GM-CSF) is undergoing clinical trials, and a vaccine was recently developed against the mesenchymal-associated transcription factor brachyury, which is currently undergoing clinical trials for the treatment of solid tumors [85]. It might be the first vaccine platform aimed at targeting a driver of tumor EMT that has successfully reached the clinical stage.

In pancreatic cancer cells, the EMT-TF SNAIL is associated with antiapoptotic features and chemoresistance [86] against 5-fluorouracil (5-FU) and gemcitabine [87]. To verify these findings, KPC, KPC; Snail ${ }^{\mathrm{cKO}}$ and KPC; Twist ${ }^{\mathrm{CKO}}$ mice were treated with gemcitabine. As expected, the latter two groups showed improved histopathology, increased survival, and defective tumor progression, as detected by magnetic resonance imaging (MRI) [13]. Equilibrative nucleoside transporter (ENT1) and concentrating nucleoside transporter (Cnt3) were significantly upregulated in cancer cells compared with KPC cells, which might constitute the mechanism connecting EMT and chemoresistance in PDAC. Regardless, the crucial role of ZEB1 in pancreatic cell drug resistance has been demonstrated in numerous studies, and EMT inducers including Twist and SNAIL confer antiapoptosis properties $[88,89]$. Following treatment with gemcitabine in ZEB1-knockdown cells, proliferation was strongly repressed, though the proliferative capacities of both control and ZEB1knockdown clusters were similar without drug treatment [25]. Additionally, in assessing the correlation of E-cadherin expression, morphological changes were identified by light microscopy [77], with E-cadherin and ZEB1 expression showing inverse correlations. Loss of E-cadherin expression mediated by transcriptional suppression has been associated with a poor clinical prognosis in several types of cancers $[90,91]$. Interestingly, as mentioned above, EMT is lethal in TGF- $\beta$-sensitive PDA cells because TGF $\beta$-induced Sox 4 changes from an inducer of tumorigenesis to a promoter of apoptosis. Although they can also lead to chemoresistance, TGF- $\beta$ inhibitors are the most intensively investigated antiEMT compounds for use as anticancer agents.

\section{Conclusion and Future Perspectives}

Tremendous efforts and notable achievements have been made in the past few decades; however, pancreatic cancer patient survival has not improved [1, 2, 92]. With further research on the EMT process and its regulation, these cellular programs will be associated with disease progression and therapy, especially in cancer, and will reveal potential targets for treating pancreatic cancer. Whether EMT participates in pancreatic cancer dissemination and colonization remains under debate. The role of EMT-TF-miRNA appears distinct in pancreatic cancer compared with other carcinomas, such as breast cancer, indicating the diversity of EMT function in different cancers. Thus, some essential EMT regulation network factors must be reverified in pancreatic cancer. Furthermore, EMT might be indispensable for pancreatic cancer metastasis or dissemination. Although EMT is considered the crucial event of carcinoma invasion and dissemination, due to its complex regulatory networks, direct evidence linking colonization and EMT is lacking, and further studies are needed to reveal the true association between EMT and metastasis. Additionally, according to the results of an experimental model of pancreatic cancer and premalignant lesions, these programs might occur at an early stage of carcinoma, leading us to consider that EMT may be a crucial prognostic indicator. A quantitative EMT scoring system for highlighting the developmental lineage of each cancer type defined by both a micro- and macro-EMT gradient was recently established based on gene expression profiles, ranking the EMT state from -1.0 to +1.0 [43]. Additionally, each cancer stage shows a distinct propensity for diverse EMT states.

In pancreatic cancer, CPCs are crucial for both dissemination and stemness. CPCs derived from primary lesion cells intravasate into the circulation and acquire self-renewal and differentiation traits through EMT. Further probing for CPCs in colonization, tumorigenesis, and dissemination is thought to be an EMT breakthrough and unique to the progression of pancreatic tumors. Due to their emergence in premalignant lesions, CPCs in the circulation could be prognostic or diagnostic factors of the tumor process as well 
as a target of anti-EMT agents. The interactions of EMT with the inflammation microenvironment are worth further exploration.

EMT is proposed to be a suppressor of cancer cell proliferation, drug transporters, and concentrating proteins, all of which are involved in the chemoresistance of pancreatic cancer. Due to the tight association between chemoresistance and EMT, traditional chemotherapy drugs such as gemcitabine could be coadministered with an anti-EMT agent or a drug that reverses the EMT process. The emergence of personalized therapy strategies and recent advances in pharmacogenomics will provide distinct opportunities for individualized cancer treatment strategies. Indeed, the diversity of the EMT network in different individuals and different subpopulations and even at different stages of tumor development precisely demands individualized treatment strategies. The EMT pathway and transitional events can be implemented to target tumors and enhance current treatment strategies.

\section{Consent}

Informed consent was obtained from all individual participants included in the study.

\section{Conflicts of Interest}

The authors declare that they have no conflicts of interest.

\section{Authors' Contributions}

Shuai Wang and Shuai Huang contributed equally to this work.

\section{Acknowledgments}

The authors are grateful for the colleagues who provided suggestions regarding this manuscript and the pioneer researchers who provided the data in the references.

\section{References}

[1] R. L. Siegel, K. D. Miller, and A. Jemal, "Cancer statistics, 2016," CA: A Cancer Journal for Clinicians, vol. 66, no. 1, pp. 7-30, 2016.

[2] C. L. Wolfgang, J. M. Herman, D. A. Laheru et al., "Recent progress in pancreatic cancer," CA: A Cancer Journal for Clinicians, vol. 63, no. 5, pp. 318-348, 2013.

[3] J. Long, G.-P. Luo, Z.-W. Xiao et al., "Cancer statistics: Current diagnosis and treatment of pancreatic cancer in Shanghai, China," Cancer Letters, vol. 346, no. 2, pp. 273-277, 2014.

[4] P. P. Provenzano, C. Cuevas, A. E. Chang, V. K. Goel, D. D. von Hoff, and S. R. Hingorani, "Enzymatic targeting of the stroma ablates physical barriers to treatment of pancreatic ductal adenocarcinoma," Cancer Cell, vol. 21, no. 3, pp. 418-429, 2012.

[5] A. Stathis and M. J. Moore, "Advanced pancreatic carcinoma: current treatment and future challenges," Nature Reviews Clinical Oncology, vol. 7, no. 3, pp. 163-172, 2010.
[6] A. D. Rhim, E. T. Mirek, N. M. Aiello et al., "EMT and dissemination precede pancreatic tumor formation," Cell, vol. 148, no. 1-2, pp. 349-361, 2012.

[7] M. K. Jolly, S. C. Tripathi, D. Jia et al., "Stability of the hybrid epithelial/mesenchymal phenotype," Oncotarget, vol. 7, no. 19, pp. 27067-27084, 2016.

[8] G. K. Alderton, "Metastasis: epithelial to mesenchymal and back again," Nature Reviews Cancer, vol. 13, no. 1, article 3, 2013.

[9] W. Li and Y. Kang, "Probing the Fifty Shades of EMT in Metastasis," Trends in Cancer, vol. 2, no. 2, pp. 65-67, 2016.

[10] B. L. Khoo, S. C. Lee, P. Kumar et al., "Short-term expansion of breast circulating cancer cells predicts response to anti-cancer therapy," Oncotarget , vol. 6, no. 17, pp. 15578-15593, 2015.

[11] M. Yu, A. Bardia, B. S. Wittner et al., "Circulating breast tumor cells exhibit dynamic changes in epithelial and mesenchymal composition," Science, vol. 339, no. 6119, pp. 580-584, 2013.

[12] J. P. Thiery and C. T. Lim, "Tumor dissemination: An EMT affair," Cancer Cell, vol. 23, no. 3, pp. 272-273, 2013.

[13] X. Zheng, J. L. Carstens, J. Kim et al., "Epithelial-tomesenchymal transition is dispensable for metastasis but induces chemoresistance in pancreatic cancer," Nature, vol. 527, no. 7579, pp. 525-530, 2015.

[14] T. Brabletz, A. Jung, S. Reu et al., "Variable $\beta$-catenin expression in colorectal cancers indicates tumor progression driven by the tumor environment," Proceedings of the National Acadamy of Sciences of the United States of America, vol. 98, no. 18, pp. 10356$10361,2001$.

[15] R. Y.-J. Huang, M. K. Wong, and T. Z. Tan, "An EMT spectrum defines an anoikis-resistant and spheroidogenic intermediate mesenchymal state that is sensitive to e-cadherin restoration by a src-kinase inhibitor, saracatinib (AZD0530)," Cell Death of Disease, vol. 4, article e915, 2013.

[16] T. Brabletz, A. Jung, S. Spaderna, F. Hlubek, and T. Kirchner, "Migrating cancer stem cells-an integrated concept of malignant tumour progression," Nature Reviews Cancer, vol. 5, no. 9, pp. 744-749, 2005.

[17] A. Díaz-López, G. Moreno-Bueno, and A. Cano, "Role of microRNA in epithelial to mesenchymal transition and metastasis and clinical perspectives," Cancer Management and Research, vol. 6, no. 1, pp. 205-216, 2014.

[18] S. Lamouille, J. Xu, and R. Derynck, "Molecular mechanisms of epithelial-mesenchymal transition," Nature Reviews Molecular Cell Biology, vol. 15, no. 3, pp. 178-196, 2014.

[19] C. C. Warzecha and R. P. Carstens, "Complex changes in alternative pre-mRNA splicing play a central role in the epithelialto-mesenchymal transition (EMT)," Seminars in Cancer Biology, vol. 22, no. 5-6, pp. 417-427, 2012.

[20] M. Pickup, S. Novitskiy, and H. L. Moses, “The roles of TGF $\beta$ in the tumour microenvironment," Nature Reviews Cancer, vol. 13, no. 11, pp. 788-799, 2013.

[21] A. Puisieux, T. Brabletz, and J. Caramel, "Oncogenic roles of EMT-inducing transcription factors," Nature Cell Biology, vol. 16, no. 6, pp. 488-494, 2014.

[22] M. A. Nieto, R.-J. Huang, R. Jackson, and J. Thiery, "EMT: 2016," Cell, vol. 166, no. 1, pp. 21-45, 2016.

[23] J. Yang and R. A. Weinberg, "Epithelial- mesenchymal transition: at the crossroads of development and tumor metastasis," Developmental Cell, vol. 14, no. 6, pp. 818-829, 2008.

[24] K. Miyazono, "Transforming growth factor- $\beta$ signaling in epithelial-mesenchymal transition and progression of cancer," Proceedings of the Japan Academy Series B: Physical and Biological Sciences, vol. 85, no. 8, pp. 314-323, 2009. 
[25] U. Wellner, J. Schubert, U. C. Burk et al., "The EMT-activator ZEB1 promotes tumorigenicity by repressing stemnessinhibiting microRNAs," Nature Cell Biology, vol. 11, no. 12, pp. 1487-1495, 2009.

[26] S.-M. Park, A. B. Gaur, E. Lengyel, and M. E. Peter, “The miR200 family determines the epithelial phenotype of cancer cells by targeting the E-cadherin repressors ZEB1 and ZEB2," Genes \& Development, vol. 22, no. 7, pp. 894-907, 2008.

[27] P. A. Gregory, A. G. Bert, E. L. Paterson et al., "The miR200 family and miR-205 regulate epithelial to mesenchymal transition by targeting ZEB1 and SIP1," Nature Cell Biology, vol. 10, no. 5, pp. 593-601, 2008.

[28] M. Korpal, B. J. Ell, F. M. Buffa et al., "Direct targeting of Sec23a by miR-200s influences cancer cell secretome and promotes metastatic colonization," Nature Medicine, vol. 17, no. 9, pp. 11011109, 2011.

[29] O. H. Ocaña, R. Córcoles, Á. Fabra et al., "Metastatic colonization requires the repression of the epithelial-mesenchymal transition inducer Prrxl," Cancer Cell, vol. 22, no. 6, pp. 709724, 2012.

[30] U. Bedi, V. K. Mishra, D. Wasilewski, C. Scheel, and S. A. Johnsen, "Epigenetic plasticity: A central regulator of epithelialtomesenchymal transition in cancer," Oncotarget, vol. 5, no. 8, pp. 2016-2029, 2014.

[31] M. Hemberger, "Genetic-epigenetic intersection in trophoblast differentiation: Implications for extraembryonic tissue function," Epigenetics, vol. 5, no. 1, pp. 24-29, 2010.

[32] M. Hemberger, "Epigenetic landscape required for placental development," Cellular and Molecular Life Sciences, vol. 64, no. 18, pp. 2422-2436, 2007.

[33] Y. Lin, Y. Wu, J. Li et al., "The SNAG domain of snaill functions as a molecular hook for recruiting lysine-specific demethylase 1," EMBO Journal, vol. 29, no. 11, pp. 1803-1816, 2010.

[34] C. Dong, Y. Wu, Y. Wang et al., "Interaction with Suv39H1 is critical for Snail-mediated E-cadherin repression in breast cancer," Oncogene, vol. 32, no. 11, pp. 1351-1362, 2013.

[35] Y. Lin, C. Dong, and B. P. Zhou, "Epigenetic regulation of EMT: The snail story," Current Pharmaceutical Design, vol. 20, no. 11, pp. 1698-1705, 2014.

[36] H. Peinado, E. Ballestar, M. Esteller, and A. Cano, "Snail mediates E-cadherin repression by the recruitment of the Sin3A/histone deacetylase 1 (HDAC1)/HDAC2 complex," Molecular and Cellular Biology, vol. 24, no. 1, pp. 306-319, 2004.

[37] J. Wang, K. Scully, X. Zhu et al., "Opposing LSD1 complexes function in developmental gene activation and repression programmes," Nature, vol. 446, no. 7138, pp. 882-887, 2007.

[38] Z. Enkhbaatar, M. Terashima, D. Oktyabri et al., "KDM5B histone demethylase controls epithelial-mesenchymal transition of cancer cells by regulating the expression of the microRNA-200 family," Cell Cycle, vol. 12, no. 13, pp. 2100-2112, 2013.

[39] A. N. Abell, N. V. Jordan, W. Huang et al., "MAP3K4/CBPregulated $\mathrm{H} 2 \mathrm{~B}$ acetylation controls epithelial-mesenchymal transition in trophoblast stem cells," Cell Stem Cell, vol. 8, no. 5, pp. 525-537, 2011.

[40] N. V. Jordan, G. L. Johnson, and A. N. Abell, "Tracking the intermediate stages of epithelial-mesenchymal transition in epithelial stem cells and cancer," Cell Cycle, vol. 10, no. 17, pp. 2865-2873, 2011.

[41] W. L. Tam and R. A. Weinberg, "The epigenetics of epithelialmesenchymal plasticity in cancer," Nature Medicine, vol. 19, no. 11, pp. 1438-1449, 2013.
[42] K. R. Fischer, A. Durrans, S. Lee et al., "Epithelial-tomesenchymal transition is not required for lung metastasis but contributes to chemoresistance," Nature, vol. 527, no. 7579, pp. 472-476, 2015.

[43] T. Z. Tan, Q. H. Miow, Y. Miki et al., "Epithelial-mesenchymal transition spectrum quantification and its efficacy in deciphering survival and drug responses of cancer patients," $E M B O$ Molecular Medicine, vol. 6, no. 10, pp. 1279-1293, 2014.

[44] S. H. Au, B. D. Storey, J. C. Moore et al., "Clusters of circulating tumor cells traverse capillary-sized vessels," Proceedings of the National Acadamy of Sciences of the United States of America, vol. 113, no. 18, pp. 4947-4952, 2016.

[45] K. Podsypanina, Y.-C. N. Du, M. Jechlinger, L. J. Beverly, D. Hambardzumyan, and H. Varmus, "Seeding and propagation of untransformed mouse mammary cells in the lung," Science, vol. 321, no. 5897, pp. 1841-1844, 2008.

[46] S. B. Krantz, M. A. Shields, S. Dangi-Garimella, D. J. Bentrem, and H. G. Munshi, "Contribution of epithelial-mesenchymal transition to pancreatic cancer progression," Cancers, vol. 2, no. 4, pp. 2084-2097, 2010.

[47] C. Scheel and R. A. Weinberg, "Phenotypic plasticity and epithelial-mesenchymal transitions in cancer and normal stem cells?" International Journal of Cancer, vol. 129, no. 10, pp. 2310 2314, 2011.

[48] A. J. Trimboli, K. Fukino, A. de Bruin et al., "Direct evidence for epithelial-mesenchymal transitions in breast cancer," Cancer Research, vol. 68, no. 3, pp. 937-945, 2008.

[49] J. Y. Park, S.-M. Hong, D. S. Klimstra, M. G. Goggins, A. Maitra, and R. H. Hruban, "Pdxl expression in pancreatic precursor lesions and neoplasms," Applied Immunohistochemistry \& Molecular Morphology, vol. 19, no. 5, pp. 444-449, 2011.

[50] J. P. Neoptolemos, D. D. Stocken, H. Friess et al., "A randomized trial of chemoradiotherapy and chemotherapy after resection of pancreatic cancer," The New England Journal of Medicine, vol. 350, no. 12, pp. 1200-1210, 2004.

[51] P. C. Hermann, S. L. Huber, T. Herrler et al., "Distinct populations of cancer stem cells determine tumor growth and metastatic activity in human pancreatic cancer," Cell Stem Cell, vol. 1, no. 3, pp. 313-323, 2007.

[52] A. Jimeno, G. Feldmann, A. Suárez-Gauthier et al., "A direct pancreatic cancer xenograft model as a platform for cancer stem cell therapeutic development," Molecular Cancer Therapeutics, vol. 8, no. 2, pp. 310-314, 2009.

[53] A. Calon, E. Lonardo, and A. Berenguer-Llergo, "Stromal gene expression defines poor-prognosis subtypes in colorectal cancer," Nature Genetics, vol. 47, pp. 320-329, 2015.

[54] Z. Yu, T. G. Pestell, M. P. Lisanti, and R. G. Pestell, "Cancer stem cells," The International Journal of Biochemistry \& Cell Biology, vol. 44, no. 12, pp. 2144-2151, 2012.

[55] X. Ye, W. L. Tam, T. Shibue et al., "Distinct EMT programs control normal mammary stem cells and tumour-initiating cells," Nature, vol. 525, no. 7568, pp. 256-260, 2015.

[56] C. Li, D. G. Heidt, P. Dalerba et al., "Identification of pancreatic cancer stem cells," Cancer Research, vol. 67, no. 3, pp. 1030-1037, 2007.

[57] W.-L. Hwang, J.-K. Jiang, S.-H. Yang et al., "MicroRNA-146a directs the symmetric division of Snail-dominant colorectal cancer stem cells," Nature Cell Biology, vol. 16, no. 3, pp. 268280, 2014.

[58] B.-Z. Qian, H. Zhang, J. Li et al., "FLT1 signaling in metastasisassociated macrophages activates an inflammatory signature 
that promotes breast cancer metastasis," The Journal of Experimental Medicine, vol. 212, no. 9, pp. 1433-1448, 2015.

[59] S. R. Nielsen, V. Quaranta, A. Linford et al., "Macrophagesecreted granulin supports pancreatic cancer metastasis by inducing liver fibrosis," Nature Cell Biology, vol. 18, no. 5, pp. 549-560, 2016.

[60] X. Liu, H. Sun, J. Qi et al., "Sequential introduction of reprogramming factors reveals a time-sensitive requirement for individual factors and a sequential EMT-MET mechanism for optimal reprogramming," Nature Cell Biology, vol. 15, no. 7, pp. 829-838, 2013.

[61] X. Gaeta, Y. Xie, and W. E. Lowry, "Sequential addition of reprogramming factors improves efficiency," Nature Cell Biology, vol. 15, no. 7, pp. 725-727, 2013.

[62] T. Celià-Terrassa, O. Meca-Cortés, F. Mateo et al., "Epithelialmesenchymal transition can suppress major attributes of human epithelial tumor-initiating cells," The Journal of Clinical Investigation, vol. 122, no. 5, pp. 1849-1868, 2012.

[63] C. J. David, Y.-H. Huang, M. Chen et al., “TGF- $\beta$ Tumor Suppression through a Lethal EMT," Cell, vol. 164, no. 5, pp. 1015-1030, 2016.

[64] S. I. Grivennikov, F. R. Greten, and M. Karin, "Immunity, Inflammation, and Cancer," Cell, vol. 140, no. 6, pp. 883-899, 2010.

[65] J. Condeelis and J. W. Pollard, "Macrophages: obligate partners for tumor cell migration, invasion, and metastasis," Cell, vol. 124, no. 2, pp. 263-266, 2006.

[66] J. B. Wyckoff, Y. Wang, E. Y. Lin et al., "Direct visualization of macrophage-assisted tumor cell intravasation in mammary tumors," Cancer Research, vol. 67, no. 6, pp. 2649-2656, 2007.

[67] C. Guerra, A. J. Schuhmacher, M. Cañamero et al., "Chronic pancreatitis is essential for induction of pancreatic ductal adenocarcinoma by K-Ras oncogenes in adult mice," Cancer Cell, vol. 11, no. 3, pp. 291-302, 2007.

[68] C. Guerra, M. Collado, C. Navas et al., "Pancreatitis-induced inflammation contributes to pancreatic cancer by inhibiting oncogene-induced senescence," Cancer Cell, vol. 19, no. 6, pp. 728-739, 2011.

[69] S. Grover and S. Syngal, "Hereditary pancreatic cancer," Gastroenterology, vol. 139, no. 4, pp. 1076-e2, 2010.

[70] Y. Wu, J. Deng, P. G. Rychahou, S. Qiu, B. M. Evers, and B. P. Zhou, "Stabilization of snail by NF-kappaB is required for inflammation-induced cell migration and invasion," Cancer Cell, vol. 15, no. 5, pp. 416-428, 2009.

[71] M. Karin and F. R. Greten, "NF- $\kappa$ B: linking inflammation and immunity to cancer development and progression," Nature Reviews Immunology, vol. 5, no. 10, pp. 749-759, 2005.

[72] M. Karin, "Nuclear factor- $\kappa \mathrm{B}$ in cancer development and progression," Nature, vol. 441, no. 7092, pp. 431-436, 2006.

[73] S. Grünert, M. Jechlinger, and H. Beug, "Diverse cellular and molecular mechanisms contribute to epithelial plasticity and metastasis," Nature Reviews Molecular Cell Biology, vol. 4, no. 8, pp. 657-665, 2003.

[74] P. M. Rothwell, F. G. R. Fowkes, J. F. Belch, H. Ogawa, C. P. Warlow, and T. W. Meade, "Effect of daily aspirin on long-term risk of death due to cancer: analysis of individual patient data from randomised trials," The Lancet, vol. 377, no. 9759, pp. 3141, 2011.

[75] A. W. Lowe, M. Olsen, Y. Hao et al., "Gene expression patterns in pancreatic tumors, cells and tissues," PLoS ONE, vol. 2, no. 3, article no. e323, 2007.
[76] A. Singh and J. Settleman, "EMT, cancer stem cells and drug resistance: an emerging axis of evil in the war on cancer," Oncogene, vol. 29, no. 34, pp. 4741-4751, 2010.

[77] T. Arumugam, V. Ramachandran, K. F. Fournier et al., "Epithelial to mesenchymal transition contributes to drug resistance in pancreatic cancer," Cancer Research, vol. 69, no. 14, pp. 5820$5828,2009$.

[78] L. Eric, C. J. Yeo, K. D. Lillemoe et al., "A lethally irradiated allogeneic granulocyte-macrophage colony stimulating factorsecreting tumor vaccine for pancreatic adenocarcinoma: A phase II trial of safety, efficacy, and immune activation," Annals of Surgery, vol. 253, no. 2, pp. 328-335, 2011.

[79] G. L. Beatty, E. G. Chiorean, M. P. Fishman et al., “CD40 agonists alter tumor stroma and show efficacy against pancreatic carcinoma in mice and humans," Science, vol. 331, no. 6024, pp. 1612-1616, 2011.

[80] I. Akalay, B. Janji, M. Hasmim et al., "Epithelial-tomesenchymal transition and autophagy induction in breast carcinoma promote escape from t-cell-mediated lysis," Cancer Research, vol. 73, no. 8, pp. 2418-2427, 2013.

[81] C. Kudo-Saito, H. Shirako, T. Takeuchi, and Y. Kawakami, "Cancer metastasis is accelerated through immunosuppression during Snail-induced EMT of cancer cells," Cancer Cell, vol. 15, no. 3, pp. 195-206, 2009.

[82] M. Hölzel, A. Bovier, and T. Tüting, "Plasticity of tumour and immune cells: A source of heterogeneity and a cause for therapy resistance?" Nature Reviews Cancer, vol. 13, no. 5, pp. 365-376, 2013.

[83] N. McGranahan and C. Swanton, "Biological and therapeutic impact of intratumor heterogeneity in cancer evolution," Cancer Cell, vol. 27, no. 1, pp. 15-26, 2015.

[84] R. Basu, B. M. Whitlock, J. Husson et al., "Cytotoxic T cells use mechanical force to potentiate target cell killing," Cell, vol. 165, no. 1, pp. 100-110, 2016.

[85] D. H. Hamilton, M. T. Litzinger, A. Jales et al., "Immunological targeting of tumor cells undergoing an epithelialmesenchymal transition via a recombinant brachyury-yeast vaccine," Oncotarget, vol. 4, no. 10, pp. 1777-1790, 2013.

[86] K. Zhang, X. Jiao, X. Liu, and et al., "Knockdown of snail sensitizes pancreatic cancer cells to chemotherapeutic agents and irradiation," International Journal of Molecular Sciences, vol. 11, no. 12, pp. 4891-4892, 2010.

[87] T. Yin, C. Wang, T. Liu, G. Zhao, Y. Zha, and M. Yang, "Expression of snail in pancreatic cancer promotes metastasis and chemoresistance," Journal of Surgical Research, vol. 141, no. 2, pp. 196-203, 2007.

[88] S. Vega, A. V. Morales, O. H. Ocaña, F. Valdés, I. Fabregat, and M. A. Nieto, "Snail blocks the cell cycle and confers resistance to cell death," Genes \& Development, vol. 18, no. 10, pp. 1131-1143, 2004.

[89] Q.-Q. Li, J.-D. Xu, W.-J. Wang et al., “Twistl-mediated adriamycin-induced epithelial-mesenchymal transition relates to multidrug resistance and invasive potential in breast cancer cells," Clinical Cancer Research, vol. 15, no. 8, pp. 2657-2665, 2009.

[90] C. Peña, J. M. Garciá, J. Silva et al., "E-cadherin and vitamin D receptor regulation by SNAIL and ZEB1 in colon cancer: clinicopathological correlations," Human Molecular Genetics, vol. 14, no. 22, pp. 3361-3370, 2005.

[91] M. Dohadwala, S. Yang, J. Luo et al., "Cyclooxygenase2-dependent regulation of E-cadherin: prostaglandin $\mathrm{E}(2)$ 
induces transcriptional repressors ZEB1 and snail in non-small cell lung cancer," Cancer Research, vol. 66, no. 10, pp. 5338-5345, 2006.

[92] H. Cao, D. Le, and L.-X. Yang, "Current status in chemotherapy for advanced pancreatic adenocarcinoma," Anticancer Reseach, vol. 33, no. 5, pp. 1785-1791, 2013. 


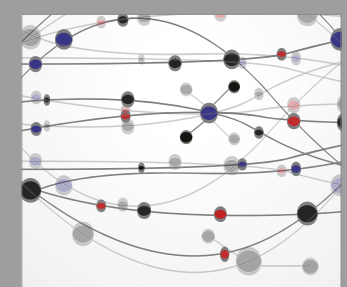

The Scientific World Journal
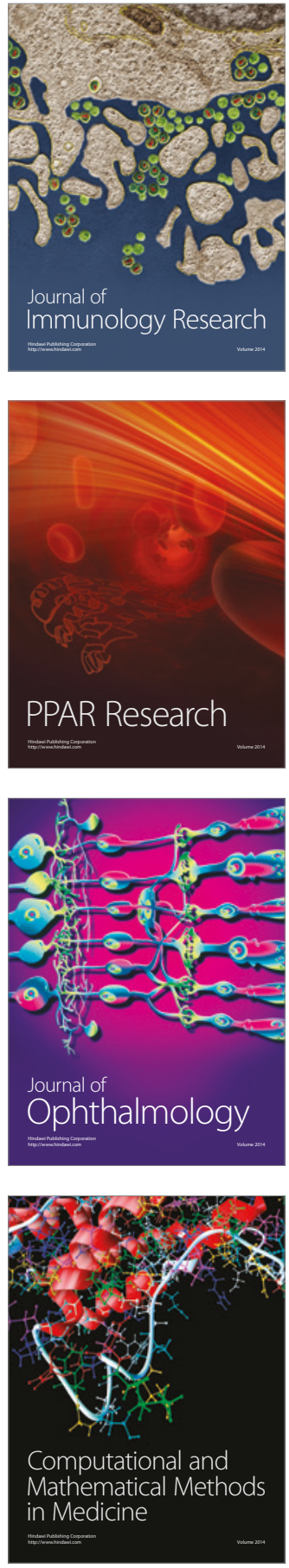

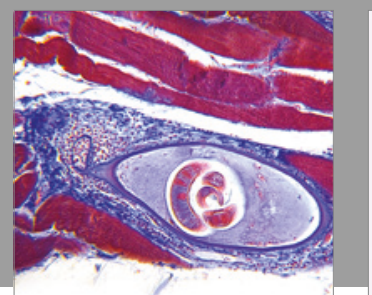

Gastroenterology Research and Practice
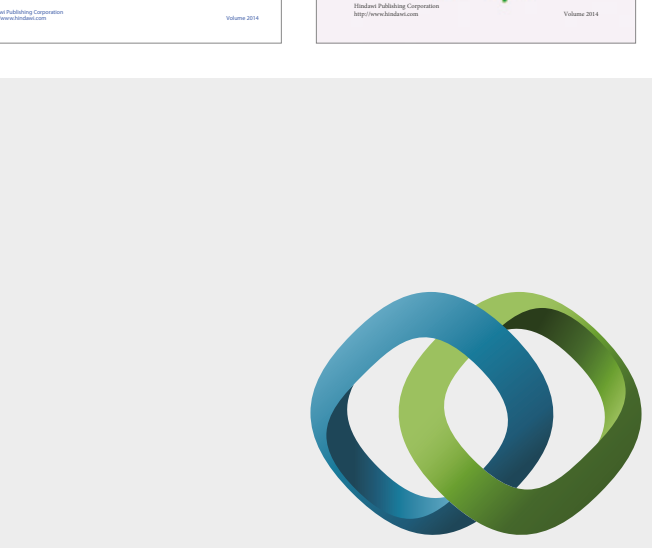

\section{Hindawi}

Submit your manuscripts at

https://www.hindawi.com
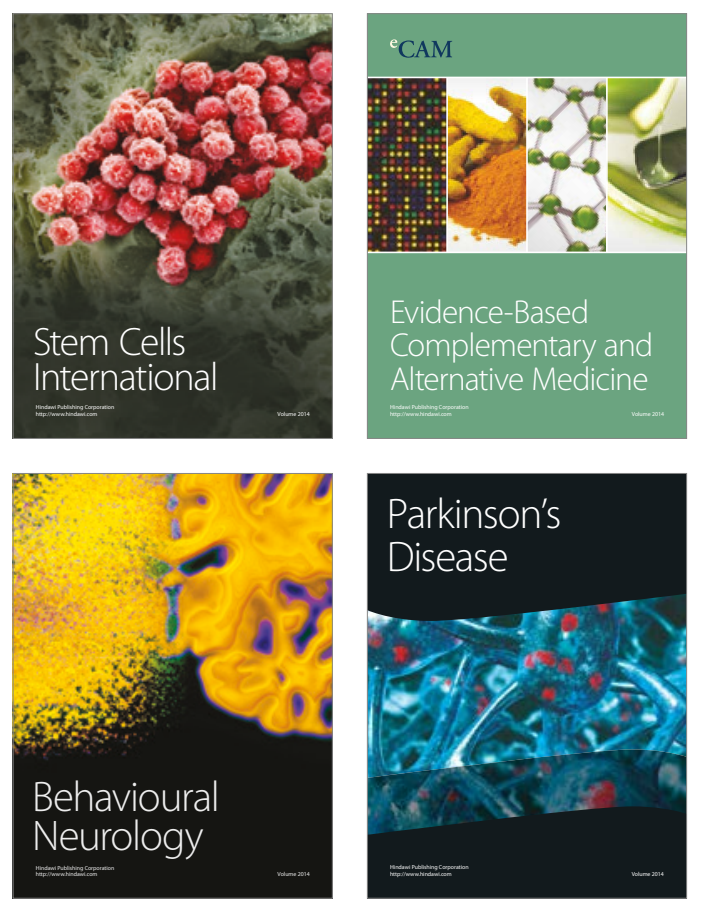
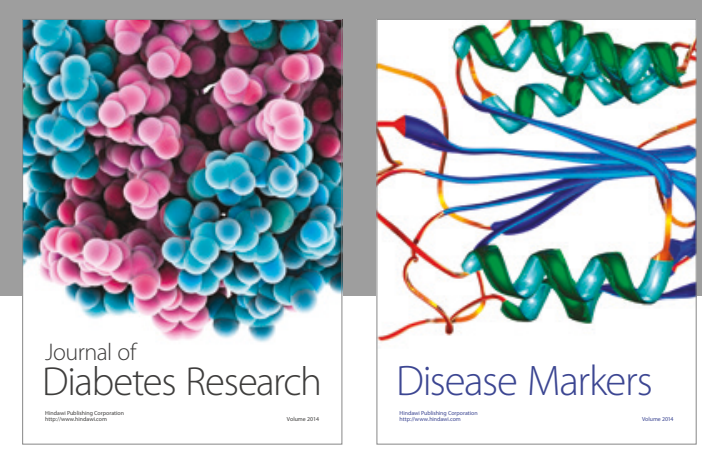

Disease Markers
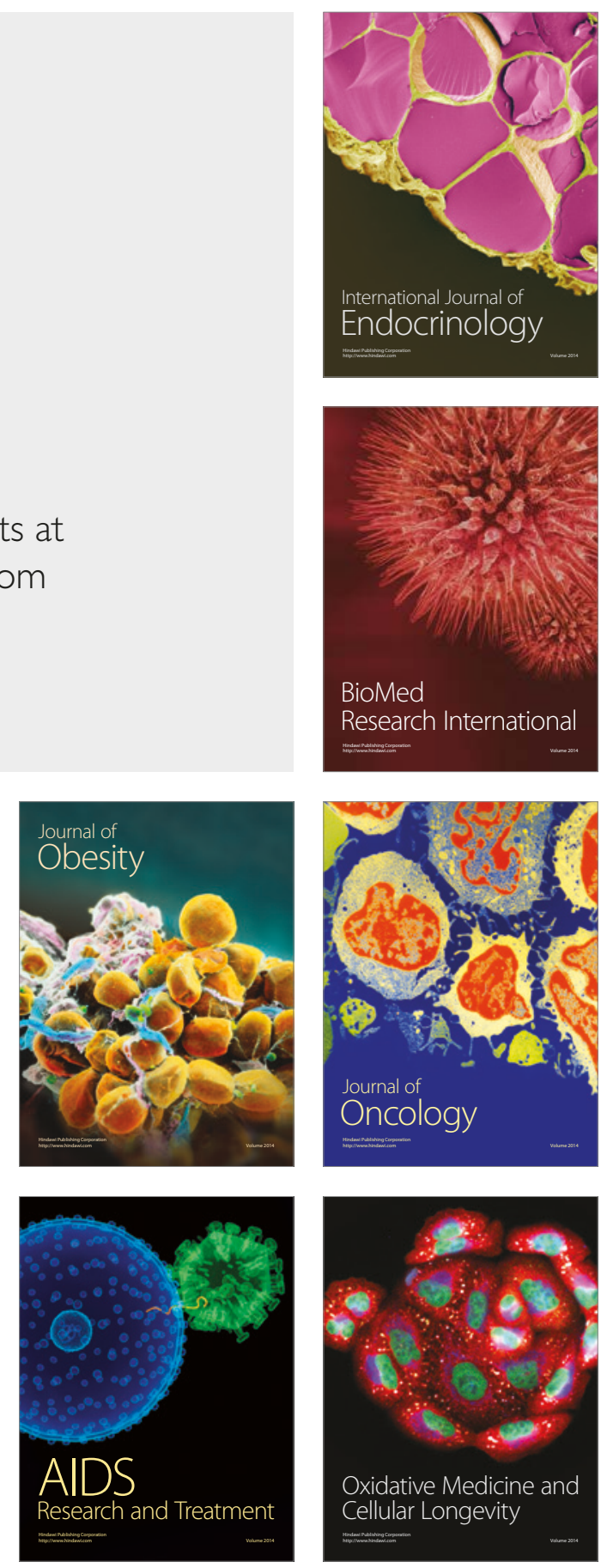